\title{
Oral estrogen leads to falsely low concentrations of estradiol in a common immunoassay
} \author{
Katherine L Imborek ${ }^{6}$, Zil Goldstein ${ }^{7,8}$ and Dina N Greene ${ }^{9,10}$ \\ 'Department of Pharmacy, University of Washington, Seattle, Washington, USA \\ ${ }^{2}$ Department of Pathology and Laboratory Medicine, University of California Irvine, Orange, California, USA \\ ${ }^{3}$ Department of Laboratories, Seattle Children's Hospital, Seattle, Washington, USA \\ ${ }^{4}$ Department of Pathology, University of lowa Hospitals and Clinics, lowa City, lowa, USA \\ IInternal Medicine, Capitol Hill Medical, Seattle, Washington, USA \\ ${ }^{6}$ Department of Family Medicine, University of lowa Hospitals and Clinics, lowa City, lowa, USA \\ ${ }^{7}$ Department of Medicine, Callen-Lorde Community Health Center, New York, New York, USA \\ ${ }^{8}$ CUNY Graduate School of Public Health and Health Policy, New York, New York, USA \\ 'Washington Kaiser Permanente, Renton, Washington, USA \\ ${ }^{10}$ Department of Laboratory Medicine and Pathology, University of Washington, Seattle, Washington, USA
}

Lauren R Cirrincione ${ }^{\mathbb{1} 1}$, Bridgit O Crews ${ }^{2}$, Jane A Dickerson ${ }^{3}$, Matthew D Krasowski ${ }^{4}$, Jessica Rongitsch ${ }^{5}$,

Correspondence should be addressed to D N Greene: dngreene@uw.edu

\begin{abstract}
Objectives: Recently, an estradiol immunoassay manufacturer (Beckman Coulter, USA) issued an 'important product notice' alerting clinical laboratories that their assay (Access Sensitive Estradiol) was not indicated for patients undergoing exogenous estradiol treatment. The objective of this analysis was to evaluate immunoassay bias relative to liquid chromatography tandem mass spectrometry (LC-MS/MS) in transgender women and to examine the influence of unconjugated estrone on measurements.

Design: Cross-sectional secondary analysis.

Methods: Estradiol concentrations from 89 transgender women were determined by 3 immunoassays (Access Sensitive Estradiol ('New BC') and Access Estradiol assays ('Old BC'), Beckman Coulter; Estradiol III assay ('Roche'), Roche Diagnostics) and LC-MS/MS. Bias was evaluated with and without adjustment for estrone concentrations. The number of participants who shifted between three estradiol concentration ranges for each immunoassay vs LC-MS/MS (>300 pg/mL, $70-300 \mathrm{pg} / \mathrm{mL}$, and $<70 \mathrm{pg} / \mathrm{mL}$ ) was calculated. Results: The New BC assay had the largest magnitude overall bias (median: $-34 \%$ ) and was $-40 \%,-22 \%$, and $-10 \%$, among participants receiving tablet, patch, or injection preparations, respectively. Overall bias was $-12 \%$ and $+17 \%$ for the Roche and Old BC assays, respectively. When measured with the New BC assay, 18 participants shifted to a lower estradiol concentration range (vs 9 and 10 participants based on Roche or Old BC assays, respectively). Adjustment for estrone did not minimize bias.

Conclusions: Immunoassay measurement of estradiol in transgender women may lead to falsely decreased concentrations that have the potential to affect management. A multidisciplinary health care approach is needed to ensure if appropriate analytical methods are available.
\end{abstract}




\section{Introduction}

Estradiol (17 $\beta$-estradiol) is a natural sex steroid available in several exogenous preparations. The Endocrine Society recommended high-dose exogenous estradiol treatment as one part of feminizing hormone therapy for transgender women (1) - people with a female gender identity who were assigned male at birth. The Endocrine Society and World Professional Association for Transgender Health (WPATH), two international transgender health-focused professional organizations, recommended clinical laboratory monitoring of serum estradiol concentrations during the first year of feminizing hormone therapy $(1,2,3)$. Despite these recommendations, neither organization commented on best practices or established challenges associated with determining exogenous estradiol concentrations in clinical settings (4).

Clinicians and laboratory scientists typically determine endogenous estradiol concentrations using commercial immunoassays due to ease of use, rapid result turnaround time, and affordability (5). Despite these advantages, early reports established cross-reactivity between estrogenic metabolites (including unconjugated and conjugated estrone, a weak estrogen) and anti-estradiol antibodies used in immunoassays (4). On April 7, 2021, a manufacturer issued an important product notice alerting customers that a widely used estradiol immunoassay was not indicated for people undergoing exogenous estradiol treatment (Access Sensitive Estradiol, Beckman Coulter, Brea, CA, USA). A related US Food and Drug Administration Medical Device Report documented an occurrence of low measured estradiol concentrations in a patient undergoing exogenous estradiol treatment during an ovarian stimulation protocol (6). Further discussion with the vendor indicated that supraphysiologic circulating estrone concentrations, a metabolite of exogenous estradiol treatment $(7,8)$, may interfere with estradiol detection (Beckman Coulter Incorporated, personal communication).

We previously reported 10- to 12-fold higher estrone concentrations among transgender women taking exogenous estradiol tablet preparations compared with transgender women taking non-oral estradiol preparations (patch or injection) $(9,10)$. To examine the effect of unconjugated estrone concentrations on estradiol immunoassay interference, we determined analytical bias relative to liquid chromatography tandem mass spectrometry (LC-MS/MS) in three estradiol commercial immunoassays among transgender women undergoing feminizing hormone therapy with tablet and non-oral estradiol preparations.

\section{Materials and methods}

\section{Samples}

We prospectively collected single whole blood specimens (5-mL gold-top serum separator tube) from a cohort of transgender women between 2017 and 2018 at two US lesbian, gay, bisexual, transgender, queer (LGBTQ)-focused clinics to determine estradiol and estrone concentrations, as described previously $(9,10)$. Among 89 participants with available samples for this analysis, all participants underwent at least 12 months of feminizing hormone therapy based on clinical need with estradiol tablets $(n=51$, total amount (range): $2-8 \mathrm{mg}$ daily), patches ( $n=9$, total amount (range): 100-200 $\mu$ g daily), or injections ( $n=29$, total amount (range): 3-10 mg weekly) at time of specimen collection $(1,10)$. We separated and stored serum aliquots at $-80^{\circ}$ until analysis. This study was approved by the Western Institutional Review Board and University of Iowa Institutional Review Board and all participants provided written informed consent. The study was conducted in accordance with the Declaration of Helsinki.

\section{Estradiol and estrone assays}

In this analysis, we determined serum estradiol concentrations using Access Sensitive Estradiol assay run on the DxI800 ('New BC,' Beckman Coulter) (11). We previously determined estradiol concentrations using two other commercial immunoassays as described (9): (1) Estradiol III assay run on Cobas E601 analyzer ('Roche', Roche Diagnostics $\mathrm{GmbH}$ ) and (2) Access Estradiol assay run on a DxI800 analyzer, the previous generation estradiol assay ('Old BC', Beckman Coulter). Table 1 summarizes apparent cross-reactivities of four estradiol immunoassays reported in package insert information, including three immunoassays used in the present study $(11,12,13,14)$. We previously determined serum estradiol and estrone concentrations by a LC-MS/MS method (6500 QTrap, Applied Biosystem Sciex) with linearity over calibration ranges of $4-700 \mathrm{pg} / \mathrm{mL}$ for estradiol and $10-5000 \mathrm{pg} / \mathrm{mL}$ for estrone (10).

\section{Data analysis}

We evaluated each estradiol immunoassay method vs LC-MS/MS using Bland-Altman plots (15). Because we previously observed statistically significant differences in estrone concentrations between oral and non-oral estradiol preparations (10), we evaluated bias by estradiol

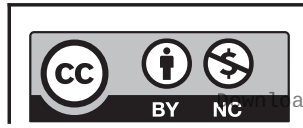

This work is licensed under a Creative Commons Attribution-NonCommercial 4.0 International License. ded from Bioscientifica.com at 04/26/2023 01:40:22AM 
Table 1 Apparent estradiol cross-reactivities reported in assay package inserts $(11,12,13,14)$.

\begin{tabular}{|c|c|}
\hline Compound $^{a}$ & $\begin{array}{l}\text { Beckman Access Estradiol } \\
\left.\text { ('Old } B C^{\prime}\right)\end{array}$ \\
\hline Estradiol & $100 \%$ \\
\hline Estrone & $1.98 \%$ \\
\hline Estrone-3-sulfate & $0.01 \%$ \\
\hline Estrone-3-glucuronide & No cross-reactivity detected \\
\hline 2-Hydroxyestrone & No data \\
\hline 4-Hydroxyestrone & No data \\
\hline $16 \alpha$-Hydroxyestrone & No data \\
\hline Estriol & $0.50 \%$ \\
\hline Ethinyl estradiol & $0.37 \%$ \\
\hline
\end{tabular}

\begin{tabular}{|c|}
\hline $\begin{array}{l}\text { Beckman Access Sensitive } \\
\left.\text { Estradiol ('New } B C^{\prime}\right)\end{array}$ \\
\hline $\begin{array}{l}100 \% \\
0.40 \% \\
0.0010 \% \\
0.0010 \% \\
\text { No data } \\
\text { No data } \\
\text { No data } \\
0.050 \% \\
0.030 \%\end{array}$ \\
\hline
\end{tabular}

\begin{tabular}{l}
\hline Roche Estradiol II \\
\hline $100 \%$ \\
$0.811 \%$ \\
$0.006 \%$ \\
$0.002 \%$ \\
No data \\
No data \\
No data \\
$0.218 \%$ \\
$0.231 \%$
\end{tabular}

\begin{tabular}{l} 
Roche Estradiol III \\
\hline $100 \%$ \\
$0.757 \%$ \\
$0.002 \%$ \\
$0.003 \%$ \\
No data \\
$0.754 \%$ \\
No data \\
$0.233 \%$ \\
$0.334 \%$
\end{tabular}

Roche Estradiol II was not utilized in the present study but was included for comparison of cross-reactivity. aEstrone is the main metabolite of estradiol. Other compounds (except ethinyl estradiol) are additional estrone metabolites. Ethinyl estradiol is a synthetic estrogen common in oral contraceptives but not used in feminizing hormone therapy.

preparation (tablet, patch, and injection). We tested data normality using the Shapiro-Wilk test $(P>0.05)$ and performed non-parametric Kruskal-Wallis one-way ANOVA for all four estradiol assay methods with Dunn's test for multiple comparisons. We summarized continuous variables as medians and interquartile ranges. A two-sided $P$-value $<0.05$ was considered statistically significant. Statistical analysis was performed using SAS software (SAS Institute Inc., Cary, NC, USA).

To associate inaccuracies in immunoassay-measured estradiol concentrations with potential changes in clinical management of feminizing hormone therapy (i.e., estradiol dose adjustment), we grouped LC-MS/MS estradiol results according to concentration ranges designated conservatively as 'supraphysiologic' (>300 pg/mL), 'within desired range' (70-300 pg/mL), and 'sub-physiologic' (<70 pg/mL). We selected these estradiol concentration ranges using a combined approach of empirical guideline-based ranges (1), prospectively derived estradiol concentration ranges determined previously within this cohort (9), and consensus among gender care providers in the community.

\section{Results}

Overall, the New BC assay demonstrated median bias of $-34 \%$ relative to LC-MS/MS ( $n=89$; Fig. $1 \mathrm{~A})$. We observed the greatest magnitude of bias within the tablet subgroup ( $n=51$, median: $-40 \%$ ) relative to the patch or injection subgroups ( $n=9,-22 \%$ and $n=29,-10 \%$, respectively). When considering the influence of estrone concentrations, the magnitude of bias in measured estradiol concentrations appeared to increase as estrone concentrations increased (Fig. 1D) but was constant when estrone concentration was plotted relative to estradiol concentration (estrone/ estradiol ratio) (Fig. 2A).

For the Roche assay, we observed median overall bias of $-12 \%$. Within the tablet, patch, or injection subgroups, median bias was $-14 \%,-13 \%$, and $-3 \%$ (Fig. 1B). Bias was relatively constant across a range of estradiol concentrations (Fig. 1E) and estrone/estradiol ratios (Fig. 2B). For the Old BC assay, we observed median overall bias of $+17 \%$ $(n=88)$ relative to LC-MS/MS. Similar to the New BC assay, we observed the greatest magnitude of bias within the tablet subgroup (median: $+23 \%$ ) relative to the patch or injection subgroups $(-17 \%, n=8$ and $+8 \%$, respectively) (Fig. 1C). Bias increased with increasing estrone concentration (Fig. 1F) but was relatively constant across a range of estrone/estradiol ratios (Fig. 2C).

When comparing measured estradiol concentrations among all estradiol assay methods, participants taking estradiol tablets had statistically significantly lower median estradiol concentrations using the New BC assay compared with LC-MS/MS assay (90 pg/mL vs 152 pg/mL, respectively, $P<0.0001$ ) (Supplementary Table 1 , see section on supplementary materials given at the end of this article) $(11,12,13,14)$. New BC median estradiol concentrations were statistically significantly lower compared with the Old BC assay (201 pg/mL, $P<0.001)$ and Roche assay (132 $\mathrm{pg} / \mathrm{mL}, P=0.0001)$. Among participants taking estradiol patch or injection, estradiol concentrations were similar between LC-MS/MS and all three estradiol immunoassays (overall $P$ values: patch, $P=0.6852$; injection, $P=0.4200$ ).

Using the New BC assay, 71 (of 89, 79.8\%) of measured estradiol concentrations did not shift between concentration groups relative to LC-MS/MS (Fig. 3A). In the $>300 \mathrm{pg} / \mathrm{mL}$ concentration group, seven participants shifted to the lower adjacent concentration group (70$300 \mathrm{pg} / \mathrm{mL}$ ). In the $70-300 \mathrm{pg} / \mathrm{mL}$ group, 11 participants

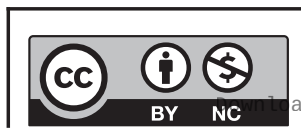

This work is licensed under a Creative Commons Attribution-NonCommercial 4.0 International License. ded from Bioscientifica.com at 04/26/2023 01:40:22AM 
Estradiol Preparation
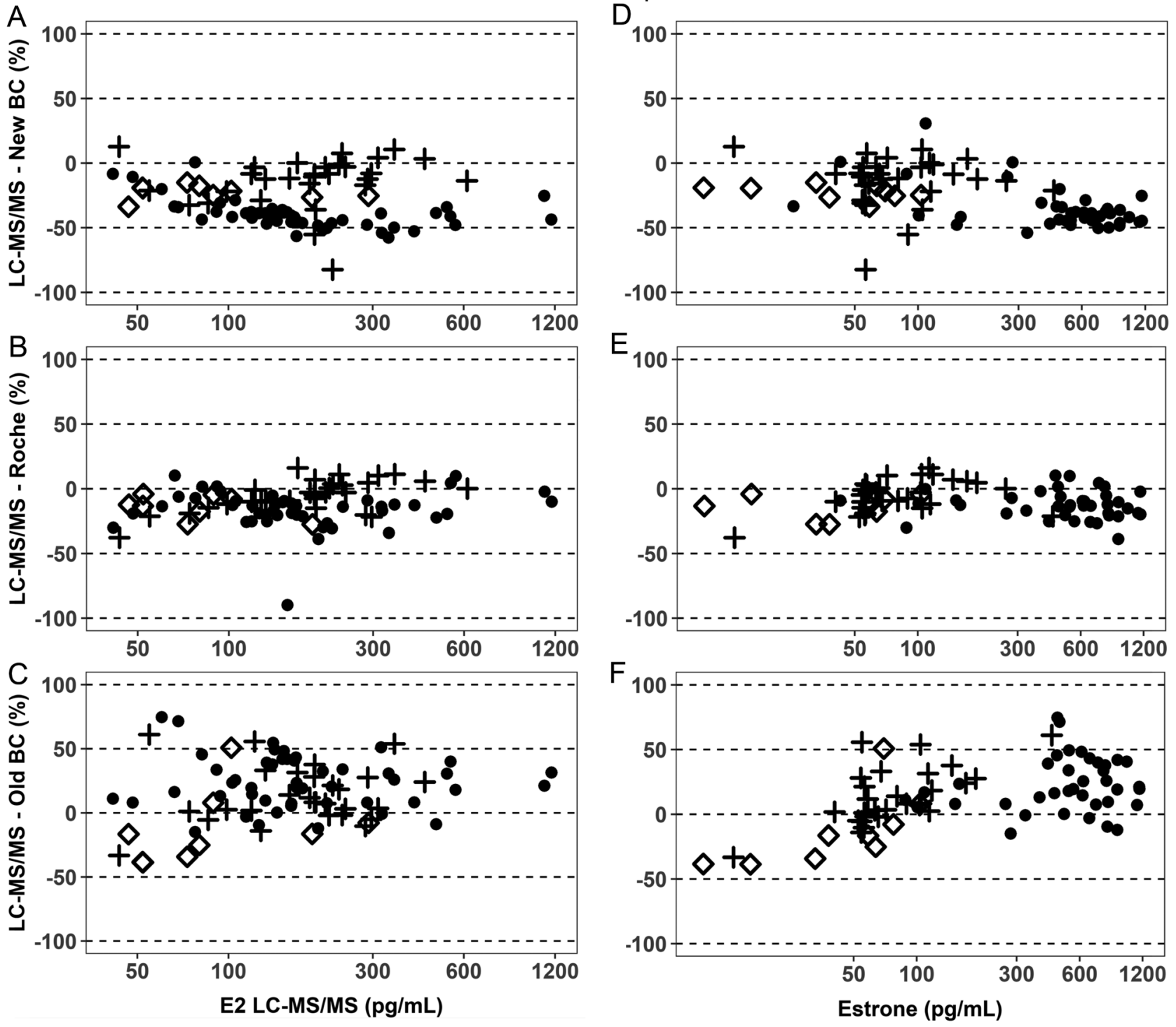

\section{+ Injection $\diamond$ Patch $\diamond$ Tablet}

Figure 1

Bland-Altman plot of percent bias between immunoassays and LC-MS/MS estradiol according to LC-MS/MS estradiol (A, B, and C) and LC-MS/MS estrone ( $D$ and $\mathrm{E}$ ) concentrations (New BC, panels A and D; Roche, panels B and E; Old BC, panels C and F).

shifted to the lower adjacent concentration group based on the New BC assay $(<70 \mathrm{pg} / \mathrm{mL})$.

Using the Roche assay, 79 (of $88,89.8 \%$ ) of measured estradiol concentrations did not shift concentration groups relative to LC-MS/MS (Fig. 3B). In the $>300 \mathrm{pg} / \mathrm{mL}$ concentration group, three participants shifted to the lower adjacent concentration group (70-300 pg/mL). In the $70--300 \mathrm{pg} / \mathrm{mL}$ group, four participants shifted to the lower adjacent concentration group $(<70 \mathrm{pg} / \mathrm{mL})$, whereas one participant shifted to the higher concentration group.
In the $<70 \mathrm{pg} / \mathrm{mL}$ concentration group, one participant shifted to the higher adjacent concentration group. Using the Old BC assay, 78 (of $88,88.6 \%$ ) of measured estradiol concentrations did not shift concentration groups relative to LC-MS/MS (Fig. 3C). In the $>300 \mathrm{pg} / \mathrm{mL}$ concentration group, no participants shifted to lower concentration groups. In the $70-300 \mathrm{pg} / \mathrm{mL}$ group, three participants shifted to the lower adjacent concentration group $(<70$ $\mathrm{pg} / \mathrm{mL}$ ), whereas three participants shifted to the higher concentration group. In the $<70 \mathrm{pg} / \mathrm{mL}$ concentration

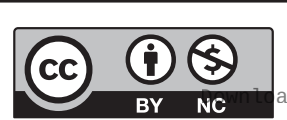



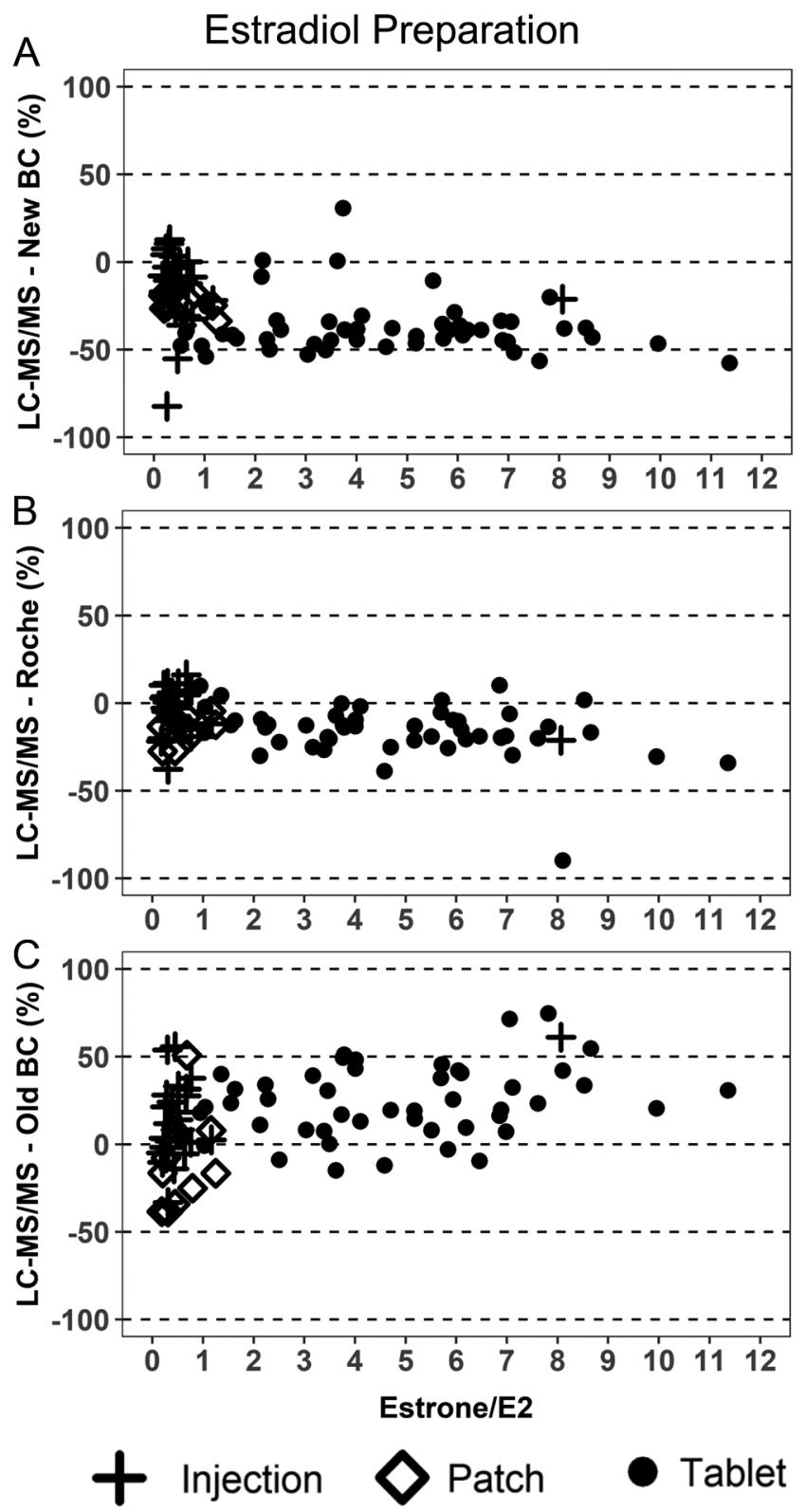

Figure 2

Bland-Altman plot of percent bias between immunoassays and LC-MS/MS according to LC-MS/MS estrone-to-estradiol ratio.

group, four participants shifted to the higher adjacent concentration group.

\section{Discussion}

We are the first investigators to evaluate bias across three estradiol immunoassays relative to LC-MS/MS in a clinical cohort of transgender women undergoing feminizing hormone therapy. Motivated by a recent manufacturer notice regarding analytical interference for estradiol immunoassays related to circulating estradiol metabolites, we observed considerable negative bias using the New BC assay (Access Sensitive Estradiol, Beckman Coulter) relative to LC-MS/MS (-34\%). The observed bias was more pronounced using the New BC assay relative to the Roche and Old BC estradiol immunoassays. When comparing estradiol concentrations between each assay method across estradiol preparations, New BC assay estradiol concentrations were statistically significantly lower compared with those measured using LC-MS/MS. These findings conflict with package insert data from 87 samples, in which New BC assay showed strong positive correlation relative to LC-MS/MS assay over estradiol concentrations 17-2119 $\mathrm{pg} / \mathrm{mL} \quad(\mathrm{r}=0.97) \quad(11)$. We recommend that clinicians and laboratory scientists determine which estradiol preparation patients are taking to facilitate measurement with a different immunoassay or via LC-MS/MS among transgender adults taking oral estradiol tablets.

Using the New BC assay, we observed nearly half of participants in the $>300 \mathrm{pg} / \mathrm{mL}$ concentration group (determined by LC-MS/MS) shifted to the 70-300 pg/mL estradiol concentration range (7 of 15 participants). Because the Endocrine Society and WPATH recommended monitoring estradiol concentrations to detect 'supraphysiologic' concentrations during feminizing hormone therapy $(1,2,3)$, clinicians may dose-decrease estradiol treatment in response to high estradiol measures. Our findings suggest that care providers using the New BC assay may not detect clinically actionable estradiol concentrations, specifically measures that warrant dosedecreased estradiol treatment. This exposure may place transgender patients at risk for estradiol exposure-related adverse events (1), although exact estradiol concentrations associated with increased risk of cardiovascular toxicities remain to be determined for transgender patients (16).

Although data are lacking for transgender women undergoing estrogen treatment, several investigators examined immunoassay performance among adults taking oral exogenous estradiol treatment for either ovarian stimulation or menopausal hormone therapy $(17,18)$. Dancoine et al. observed agreement between an automated chemiluminescent estradiol assay method (Immulite, Diagnostic Products Corp., Los Angeles, CA, USA) and RIA among 41 cisgender women undergoing exogenous estradiol treatment as part of ovarian stimulation protocols, although most participants took non-oral estradiol preparations (17). Cook et al. observed discrepant estradiol concentrations among three commercial immunoassays and a RIA method among cisgender women undergoing

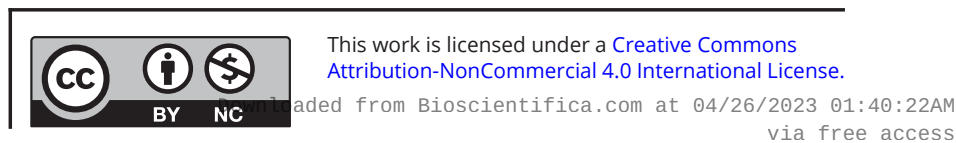




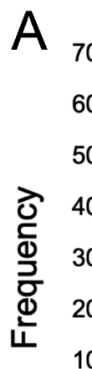

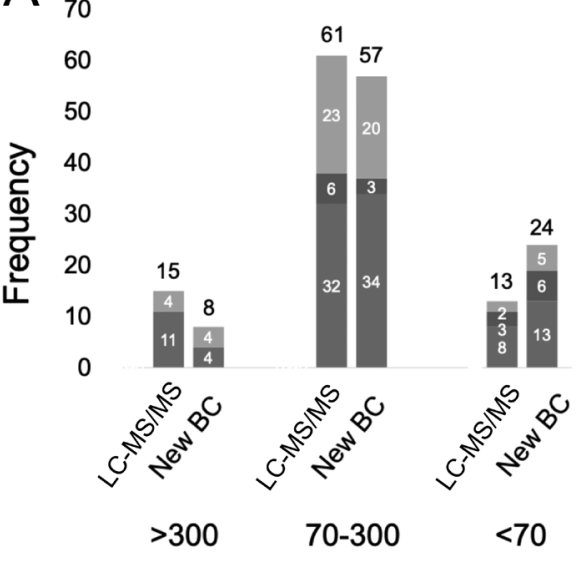

B Estradiol Preparation

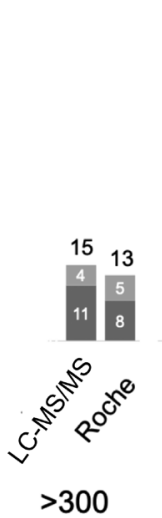

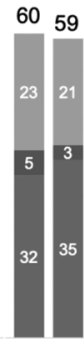

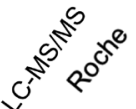

70-300
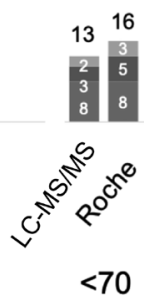

C

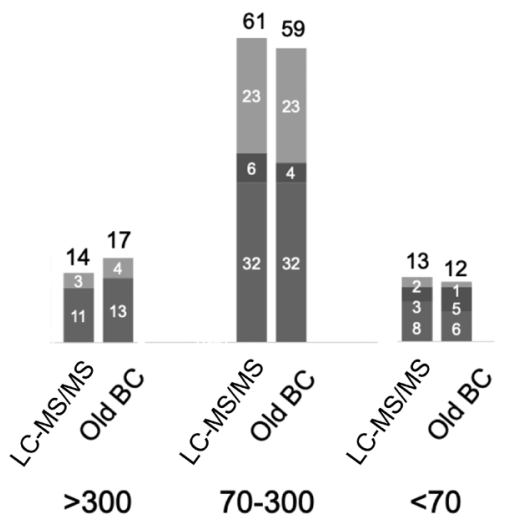

\section{Estradiol concentration $(\mathrm{pg} / \mathrm{mL})$ \\ Injection Patch Tablet}

\section{Figure 3}

Frequency of participants who shifted between estradiol concentration groups ('supraphysiologic' estradiol concentration range: $>300 \mathrm{pg} / \mathrm{mL}$; 'within desired range' estradiol concentration range: $70-300$ pg/mL; and 'sub-physiologic' estradiol concentration range: $<70$ pg/mL) based on immunoassay versus vs liquid chromatography and tandem mass spectrometry (LC-MS/MS). (A) Estradiol concentration group shifts between LC-MS/MS vs New BC. (B) Estradiol concentration group shifts between LC-MS/MS vs Roche. (C) Estradiol concentration group shifts between LC-MS/MS vs Old BC.

oral menopausal hormone therapy, although all participants were taking oral conjugated estrogens, which has established cross-reactivity with commercial estradiol immunoassay methods (18, 19). Cao et al. observed unconjugated estriol, an active estradiol metabolite that is elevated during pregnancy, led to negative interference in an estradiol microparticle enzyme immunoassay (AxSYM, Abbott Laboratories), although this finding was based on assay proficiency testing samples $(20,21)$.

We previously reported statistically significantly higher median estrone concentrations among participants taking estradiol tablets within this cohort $(693.0 \mathrm{pg} / \mathrm{mL})$ compared with those taking non-oral preparations (patch: $58.6 \mathrm{pg} / \mathrm{mL}$, injection: $67.5 \mathrm{pg} / \mathrm{mL}$, both $P<0.001$ vs tablet) (10). Other investigators similarly observed numerically higher estrone concentrations among transgender women taking oral estradiol tablets compared with estradiol patches ( $410 \mathrm{pg} / \mathrm{mL}$ (95\% CI, 347-473 pg/mL) vs $51 \mathrm{pg} / \mathrm{mL}$ (95\% CI, 41-60 pg/mL), respectively) (22). These findings are consistent with extensive first-pass metabolism of oral estradiol tablets to the metabolite estrone, which circulates predominantly as estrone-3-sulfate and is further metabolized to estrone glucuronide, 2-hydroxyestrone, 4-hydroxyestrone, 16 $\alpha$-hydroxyestrone, and estriol (21).

Typically, investigators suggest estradiol metabolites to contribute to potential cross-reactivity with assay antisera leading to positive bias in estradiol concentration measurements $(18,23)$. Immunoassay package insert cross- reactivity data predicted minimal interference by estrone and its conjugates on measured estradiol concentrations $(11,12,13,24)$. Of note, the New BC assay package insert reported lower cross-reactivity with estrone and estrone3 -sulfate compared with the Old BC assay (Table 1) (11, $12,13,14)$. This may indicate that interference was not detected by routine cross-reactivity testing protocols commonly reported in assay package inserts. Alternatively, other interferences, including well-established elevations in sex hormone-binding globulin during oral estradiol treatment (22), may lead to underestimated estradiol concentrations $(18,23)$. One limitation of this analysis is that we only measured unconjugated estrone metabolite using LC-MS/MS. Future studies should analyze a broader panel of estrone metabolites, including the conjugated and hydroxylated metabolites, and sex hormone-binding globulins to better characterize their role in estradiol assay interference among transgender adults.

Negative assay bias, as we observed in this analysis, may place patients at risk for inappropriately high estradiol dosing. The safety implications of prolonged, supratherapeutic estradiol concentrations for transgender adults are largely unknown (16), although accurate estradiol measures are an important tool for determining estradiol concentrations associated with increased venous thromboembolic risks. Despite its importance in transgender medical care, estradiol concentration determination in clinical settings is complicated 
$(25,26,27)$. Because transgender people face disparities in all aspects of health care including in clinical laboratory settings (28), accurate estradiol measurement is a crucial component of safe, equitable medical care access for transgender adults. Clinical laboratories are often siloed from other areas of the healthcare team, and laboratory scientists typically do not have immediate access to the clinical indication for serum estradiol laboratory orders. Although there is increased interest in adapting electronic medical records to include sexual orientation and gender identity data for clinical decision support (29), additional work is needed to systematically alert laboratory personnel about whether a specific immunoassay may be required for a transgender person undergoing feminizing hormone therapy.

Despite a lack of evidence to support traditional therapeutic drug monitoring of estradiol during feminizing hormone therapy, this approach appears to persist in clinical practice (30). Current transgender health-focused guides do not endorse therapeutic drug monitoring for estradiol treatment; instead, they recommend monitoring estradiol treatment for 'supraphysiologic' concentrations $(1,2,3)$, although this definition may vary depending on expert opinion and laboratory reference intervals. Importantly, no data are available to establish specific serologic data with estradiol efficacy or safety during feminizing hormone therapy (e.g. breast development and cardiovascular risks) $(16,31)$, which is essential to justify therapeutic monitoring of any drug (32). Given the potential for interference by exogenous estradiol and negative bias reported in our analysis, these factors need to be examined collectively for laboratory scientists to make informed recommendations on how to determine estradiol concentrations in clinical settings and when to dose-adjust estradiol treatment among transgender patients. As new, highly sensitive immunoassays come to the market, manufacturers and regulatory bodies need to consider the transgender population and its medical needs when evaluating new devices to ensure potential health care disparities are mitigated.

\section{Conclusion}

The Beckman Coulter Access Sensitive Estradiol assay demonstrated considerable negative bias when used among transgender women undergoing feminizing hormone therapy. Clinicians and laboratory scientists currently lack strategies to systematically select patients who may benefit from specific immunoassay or LC-MS/MS methods. Professional societies involved with transgender medicine should speak to this issue to increase awareness among clinicians and laboratory scientists.

\section{Supplementary materials}

This is linked to the online version of the paper at https://doi.org/10.1530/ EC-21-0550.

\section{Declaration of interest}

The authors declare that there is no conflict of interest that could be perceived as prejudicing the impartiality of the research reported.

\section{Funding}

This work did not receive any specific grant from any funding agency in the public, commercial or not-for-profit sector.

\section{References}

1 Hembree WC, Cohen-Kettenis PT, Gooren L, Hannema SE, Meyer WJ, Murad MH, Rosenthal SM, Safer JD, Tangpricha V \& T'Sjoen GG. Endocrine treatment of gender-dysphoric/gender-incongruent persons: an Endocrine Society* clinical practice guideline. Journal of Clinical Endocrinology and Metabolism 2017102 3869-3903. (https:// doi.org/10.1210/jc.2017-01658)

2 Feldman J \& Safer J. Hormone therapy in adults: suggested revisions to the sixth version of the standards of care. International Journal of Transgenderism 200911 146-182. (https://doi. org/10.1080/15532730903383757)

3 Coleman E, Bockting W, Botzer M, Cohen-Kettenis P, DeCuypere G, Feldman J, Fraser L, Green J, Knudson G, Meyer WJ, et al. Standards of care for the health of transsexual, transgender, and gender-nonconforming people, version 7. International Journal of Transgenderism 201213 165-232. (https://doi.org/10.1080/15532739.2 011.700873)

4 Stanczyk FZ, Cho MM, Endres DB, Morrison JL, Patel S \& Paulson RJ. Limitations of direct estradiol and testosterone immunoassay kits. Steroids 200368 1173-1178. (https://doi.org/10.1016/j. steroids.2003.08.012)

5 Stanczyk FZ \& Clarke NJ. Measurement of estradiol - challenges ahead. Journal of Clinical Endocrinology and Metabolism 2014 99 56-58. (https://doi.org/10.1210/jc.2013-2905)

6 U.S. Food and Drug Administration. Manufacturer and user facility device experience database adverse event report: Beckman Coulter access sensitive estradiol assay. (available at: https://www.accessdata. fda.gov/scripts/cdrh/cfdocs/cfMAUDE/detail.cfm?mdrfoi_ $\mathrm{id}=11614267 \& \mathrm{pc}=\mathrm{CHP}$ )

7 Karim R, Mack WJ, Hodis HN, Roy S \& Stanczyk FZ. Influence of age and obesity on serum estradiol, estrone, and sex hormone binding globulin concentrations following oral estrogen administration in postmenopausal women. Journal of Clinical Endocrinology and Metabolism 200994 4136-4143. (https://doi.org/10.1210/jc.2009-0643)

8 Lobo RA \& Cassidenti DL. Pharmacokinetics of oral 17 beta-estradiol. Journal of Reproductive Medicine 199237 77-84.

9 Greene DN, Schmidt RL, Winston McPherson G, Rongitsch J, Imborek KL, Dickerson JA, Drees JC, Humble RM, Nisly N, Dole NJ, et al. Reproductive endocrinology reference intervals for transgender women on stable hormone therapy. Journal of Applied Laboratory Medicine 20216 15-26. (https://doi.org/10.1093/jalm/jfaa028)

10 Cirrincione LR, Winston McPherson G, Rongitsch J, Sadilkova K, Drees JC, Krasowski MD, Dickerson JA \& Greene DN. Sublingual estradiol is associated with higher estrone concentrations than 
transdermal or injectable preparations in transgender women and gender nonbinary adults. LGBT Health 20218 125-132. (https://doi. org/10.1089/lgbt.2020.0249)

11 Beckman Coulter: access estradiol package insert. (available at: https:// www.beckmancoulter.com/download/phxB50134J-EN_US?type=pdf)

12 Beckman Coulter: access sensitive Estradiol ${ }^{\circledR}$ package insert. (available at: https://www.beckmancoulter.com/download/phxB84425G-EN_ US?type=pdf)

13 Roche: Elecsys ${ }^{\circledast}$ Estradiol III package insert. (available at: https://www rochecanada.com/content/dam/rochexx/roche-ca/products/docs/ package_inserts/Can\%20PI\%20Estradiol\%20III-06656021190-V6-En. pdf)

14 Roche: Elecsys ${ }^{\circledast}$ Estradiol II package insert. (available at: https://www. rochecanada.com/content/dam/rochexx/roche-ca/products/docs/ package_inserts/Can\%20PI\%20Estradiol\%20III-06656021190-V6-En.pdf)

15 Bland JM \& Altman DG. Statistical methods for assessing agreement between two methods of clinical measurement. Lancet $19861307-310$. (https://doi.org/10.1016/S0140-6736(86)90837-8)

16 Goldstein Z, Khan M, Reisman T \& Safer JD. Managing the risk of venous thromboembolism in transgender adults undergoing hormone therapy. Journal of Blood Medicine 201910 209-216. (https://doi. org/10.2147/JBM.S166780)

17 Dancoine F, Couplet G, Buvat J, Guittard C, Marcolin G \& Fourlinnie JC. Analytical and clinical evaluation of the Immulite ${ }^{\circledast}$ estradiol assay in serum from patients undergoing in vitro fertilization: estradiol increase in mature follicles. Clinical Chemistry 199743 1165-1171. (https://doi.org/10.1093/clinchem/43.7.1165)

18 Cook NJ \& Read GF. Oestradiol measurement in women on oral hormone replacement therapy: the validity of commercial test kits. British Journal of Biomedical Science 1995 52 97-101.

19 Stanczyk FZ, Jurow J \& Hsing AW. Limitations of direct immunoassays for measuring circulating estradiol levels in postmenopausal women and men in epidemiologic studies. Cancer Epidemiology, Biomarkers and Prevention 201019 903-906. (https://doi.org/10.1158/1055-9965.EPI10-0081)

20 Cao Z, Swift TA, West CA, Rosano TG \& Rej R. Immunoassay of estradiol: unanticipated suppression by unconjugated estriol. Clinical Chemistry 200450 160-165. (https://doi.org/10.1373/ clinchem.2003.023325)

$21 \mathrm{Kuhl} \mathrm{H}$. Pharmacology of estrogens and progestogens: influence of different routes of administration. Climacteric 20058 (Supplement 1) 3-63. (https://doi.org/10.1080/13697130500148875)

22 Tebbens M, Heijboer AC, T’Sjoen G, Bisschop PH \& den Heijer M. The role of estrone in feminizing hormone treatment. Journal of Clinical
Endocrinology and Metabolism 2021107 e458-e466. (https://doi. org/10.1210/clinem/dgab741)

23 Stanczyk FZ, Lee JS \& Santen RJ. Standardization of steroid hormone assays: why, how, and when? Cancer Epidemiology, Biomarkers and Prevention 200716 1713-1719. (https://doi.org/10.1158/1055-9965.EPI06-0765)

24 Krasowski MD, Drees D, Morris CS, Maakestad J, Blau JL \& Ekins S. Cross-reactivity of steroid hormone immunoassays: clinical significance and two-dimensional molecular similarity prediction. BMC Clinical Pathology 201414 33. (https://doi.org/10.1186/1472-689014-33)

25 Rosner W, Hankinson SE, Sluss PM, Vesper HW \& Wierman ME. Challenges to the measurement of estradiol: an Endocrine Society position statement. Journal of Clinical Endocrinology and Metabolism 201398 1376-1387. (https://doi.org/10.1210/jc.2012-3780)

26 Vesper HW, Botelho JC, Vidal ML, Rahmani Y, Thienpont LM \& Caudill SP. High variability in serum estradiol measurements in men and women. Steroids 201482 7-13. (https://doi.org/10.1016/j. steroids.2013.12.005)

27 Vermeulen A, Kaufman JM, Goemaere S \& Van Pottelberg I. Estradiol in elderly men. Aging Male 20025 98-102. (https://doi.org/10.1080/ tam.5.2.98.102)

28 Goldstein Z, Corneil TA \& Greene DN. When gender identity doesn't equal sex recorded at birth: the role of the laboratory in providing effective healthcare to the transgender community. Clinical Chemistry 201763 1342-1352. (https://doi.org/10.1373/ clinchem.2016.258780)

29 Cahill SR, Baker K, Deutsch MB, Keatley J \& Makadon HJ. Inclusion of sexual orientation and gender identity in stage 3 meaningful use guidelines: a huge step forward for LGBT health. LGBT Health 20163 100-102. (https://doi.org/10.1089/lgbt.2015.0136)

30 Conklin SE \& Knezevic CE. Advancements in the gold standard: measuring steroid sex hormones by mass spectrometry. Clinical Biochemistry 202082 21-32. (https://doi.org/10.1016/j. clinbiochem.2020.03.008)

31 Glintborg D, T'Sjoen G, Ravn P \& Andersen MS. Management of endocrine disease: optimal feminizing hormone treatment in transgender people. European Journal of Endocrinology 2021185 R49-R63. (https://doi.org/10.1530/EJE-21-0059)

32 Buxton ILO. Pharmacokinetics: the dynamics of drug absorption, distribution, metabolism, and elimination. In Goodman and Gilman's: The Pharmacological Basis of Therapeutics, 13th ed. Eds LL Brunton, R Hilal-Dandan \& BC Knollmann. New York, NY: McGraw-Hill Education, 2017

Received in final form 23 December 2021

Accepted 11 January 2022

Accepted Manuscript published online 11 January 2022 https://ec.bioscientifica.com https://doi.org/10.1530/EC-21-0550 (c) 2022 The authors Published by Bioscientifica Ltd

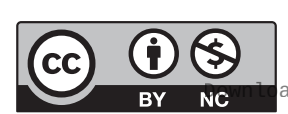

This work is licensed under a Creative Commons Attribution-NonCommercial 4.0 International License. ded from Bioscientifica.com at 04/26/2023 01:40:22AM 